\title{
Chaos and a Resonance Mechanism for Structure Formation in Inflationary Models*
}

\author{
H. P. de Oliveira ${ }^{\dagger}$ \\ Universidade do Estado do Rio de Janeiro \\ Instituto de Física - Departamento de Física Teórica, \\ CEP 20550-013 Rio de Janeiro, RJ, Brazil \\ I. Damião Soares ${ }^{\ddagger}$ \\ Centro Brasileiro de Pesquisas Fúsicas \\ Rua Dr. Xavier Sigaud, 150 \\ CEP 22290, Rio de Janeiro - RJ, Brazil
}

\begin{abstract}
We exhibit a resonance mechanism of amplification of density perturbations in inflationary models, using a minimal set of ingredients (an effective cosmological constant, a scalar field minimally coupled to the gravitational field and matter), common to most models in the literature of inflation. This mechanism is based on the structure of homoclinic cylinders, emanating from an unstable periodic orbit in the neighborhood of a saddle-center critical point, present in the phase space of the model. The cylindrical structure induces oscillatory motions of the scales of the universe whenever the orbit visits the neighborhood of the saddle-center, before the universe enters a period of exponential expansion. The oscillations of the scale functions produce, by a resonance mechanism, the amplification of a selected wave number spectrum of density perturbations, and can explain the hierarchy of scales observed in the actual universe. The transversal crossings of the homoclinic cylinders induce chaos in the dynamics of the model, a fact intimately connected to the resonance mechanism occuring immediately before the exit to inflation.
\end{abstract}

*This essay received an "honorable mention" from the Gravity Research Foundation, 1998 - Ed.

${ }^{\dagger}$ E-mail: oliveira@symbcomp.uerj.br

${ }^{\ddagger}$ E-mail: ivano@lca1.drp.cbpf.br 
The existence of an inflationary phase in the early stages of our Universe has become one of the paradigms of modern cosmology, and is now being subject to experimental verification through the crucial measurements of small scale anisotropies in the cosmic background radiation. The basic physical ingredient for this inflationary phase is the existence of a scalar field - the inflaton field, the vacuum energy of which plays the role of a cosmological constant, engendering via the gravitational dynamics an exponential expansion in the comoving scales of the universe. This phase is considered to have evolved from a pre-inflationary phase just exiting the Planck era, the matter content of which may be thought to be modeled by radiation plus a scalar field minimally coupled to the gravitational field. To be considered successful, the inflationary paradigm must accomodate models in which initial homogeneities are generated, leading to the formation of the structure present in our actual universe. In the literature of inflation, it has been claimed that small fluctuations in the scalar field may give rise to an almost scale-free spectrum of adiabatic density perturbations (the Harrison-Zeldovich spectrum), which is the standard spectrum used in explaining galaxy formation. However the number of distinct large scale objects and structures in our universe, as galaxies, cluster of galaxies, quasars, filaments and walls, is very large and the sizes of these objects form a hierarchy of scales that is not described by the flat spectrum of the initial perturbations [1]. In other words, the scale-free adiabatic perturbations appears not to be sufficient to produce the large-scale structure of the observed universe. We are therefore led to pose the following basic question: would it be possible, in the realm of the inflationary paradigm, to obtain adiabatic perturbations with a spectrum having several maxima, which would help to explain the origin of the hierarchy of scales?

Our purpose in this essay is to give a positive answer to the above question, and show that the ingredients present in most inflationary models may engender a resonance mechanism, in the beginning of, or immediately before the inflationary period, that amplifies scalar field perturbations with a selected wave number spectrum. We emphasize the simplicity of this mechanism, which deals with a minimum set of ingredients only and does not demand the introduction of further fields, fine tuning of parameters and special initial conditions. Even if the universe inflates afterwards, the relative rate of amplitudes produced after the resonance amplification would be maintained as an imprint in the initial spectrum of density fluctuations. We will also show that this resonance amplification mechanism has a fundamental connection to the chaotic behaviour of the model, and its chaotic exit to inflation [2]. Similar resonance mechanisms have been previously applied for particle production during reheating in the realm of inflationary models [3].

Let us consider the following configuration, that may constitute a simple description of a preinflationary phase of the universe in most of the inflationary models discussed in the literature. We start from a Bianchi IX model characterized by two scale factors $A(t)$ and $B(t)$, with a perfect fluid and a cosmological constant $\Lambda$ playing the role of the vacuum energy of the scalar field. For simplicity, we consider the case of dust, but the basic features are not altered for other types of perfect fluid like radiation, for instance. According with Ref. [2], the full dynamics of the models is governed by the Hamiltonian

$$
H\left(A, B, P_{A}, P_{B}\right)=\frac{P_{A} P_{B}}{4 B}-\frac{A P_{A}^{2}}{8 B^{2}}+2 A-\frac{A^{3}}{2 B^{2}}-2 \Lambda A B^{2}-E_{0}=0,
$$

where $P_{A}$ and $P_{B}$ are the momenta canonically conjugated to $A$ and $B$, respectively, and $E_{0}$ is a constant proportional to the total matter energy of the models. The associated dynamical system has one critical point $E$ of saddle-center type in the finite region of the phase space. The phase space has two critical points at infinity, corresponding to the stable and unstable de Sitter solutions, and the scale factors $A(t)$ and $B(t)$ approach the de Sitter attractor exponentially, $A=B \cong e^{\sqrt{\Lambda / 3} t}$. The dynamical system generated by (1) admits the invariant manifold $\mathcal{M}(A=B$, $\left.P_{A}=\frac{P_{B}}{2}\right)$ in which all orbits are Friedmann models with cosmological constant and dust. The critical point $E$ is contained in $\mathcal{M}$, and that the separatrices $S$ constitute boundaries between isotropic models that collapse or escape to the de Sitter configuration. The models to be considered in this essay are the ones restricted to a neighborhood of the invariant manifold $\mathcal{M}$ and can be understood as describing the early stages of inflation in which anisotropy is present, in the form of small fluctuations.

The basic point of the amplification mechanism to be discussed here is the structure of homoclinc cylinders present in the neighborhood of the saddle-center. To see this we make use of a theorem by Moser [4] which states that it is always possible to find a set of canonical variables, say $\left(q_{1}, q_{2}, p_{1}, p_{2}\right)$, such that, in a small neighborhood of a saddle-center at the origin, the Hamiltonian is separable as

$$
H\left(q_{1}, q_{2}, p_{1}, p_{2}\right)=\frac{\sqrt{\Lambda}}{2}\left(p_{2}^{2}-q_{2}^{2}\right)-\sqrt{2 \Lambda}\left(p_{1}^{2}+q_{1}^{2}\right)-E_{0}+E_{c r} \approx 0,
$$

where $E_{c r}$ is the energy associated to the saddle-center, with the two approximatte constants of motion given by the partial energies

$$
E_{\text {hyp }}=\frac{\sqrt{\Lambda}}{2}\left(p_{2}^{2}-q_{2}^{2}\right), E_{\text {rot }}=\sqrt{2 \Lambda}\left(p_{1}^{2}+q_{1}^{2}\right)
$$


The energies $E_{h y p}$ and $E_{\text {rot }}$ will be referred to as the hyperbolic motion energy and rotational motion energy of the system about $E$, respectively. The rotational motion corresponds to periodic orbits in a linear neighborhood of $E$. For zero energy of the hyperbolic motion, we have the linear stable $V_{s}$ and unstable $V_{u}$ one-dimensional manifolds. The separatrices $S$ in the invariant manifol $\mathcal{M}$ are actually the non-linear extension of $V_{s}$ and $V_{u}$. The direct product of a periodic orbit with $V_{s}$ and $V_{u}$ generates, in the linear neighborhood of $E$, the structure of stable and unstable cylinders, which coalesce into the periodic orbit for large positive and negative times, respectively [5]. A general orbit which visits the neighborhood of $E$ has an oscillatory approach to the cylinders, the closer as the energy of the hyperbolic motion goes to zero. Away from the linear neighborhood of $E$, the linear approximation is no longer valid. Higher order terms become important for the dynamics, and the non-integrability of the system results in the distortion and twisting of the cylinders. The stable cylinder and the unstable one will cross each other transversally, producing chaotic sets [2] in the neighborhood of the invariant manifold $\mathcal{M}$; orbits with initial conditions taken on these chaotic sets will be highly sensitive to small perturbations of the initial conditions. The chaotic character manifests itself physically such that a small perturbation of initial conditions taken on these sets would change an orbit from collapse to escape into the de Sitter phase, and vice-versa. Also, it is always possible to select initial conditions in these sets such that the scale factors oscillate an arbitrary fixed time before escaping to a de Sitter phase.

The physical effects related to the growing of inhomogeneous perturbations occur during the oscillatory phase when a given orbit approach $E$ before to collapse or to escape. We consider a given orbit initially in a very small sphere of initial conditions about the invariant manifold $\mathcal{M}$. In this regime the scale factors oscillate in a small region about the critical point $E$. To avoid technical dificulties concerning the perturbative formalism in anisotropic backgrounds, and motivated by the fact that in the overall dynamics of perturbations these small anisotropy fluctuations can be averaged, we introduce the average scale factor $l(t)$ of the universe defined by

$$
l=\left(A B^{2}\right)^{1 / 3} .
$$

This approximately corresponds to a closed Friedamnn-Robertson-Walker backgound with $l(t)$ as the scale factor, either in the oscillatory regime and in the inflationary phase. In a very small neighborhood of $E$ the approximate analytical solution for the average scale factor is

$$
l(t) \approx l_{0}+C_{1} e^{t / 2 l_{0}}+C_{2} e^{-t / 2 l_{0}}+\epsilon \cos (2 \omega t)
$$

where $l_{0}=1 / \sqrt{4 \Lambda}, \omega=\sqrt{2 \Lambda}$, and $\epsilon, C_{1}$ and $C_{2}$ are very small arbitrary constants of the same order of $R$. The exponential terms are associated to the hyperbolic energy mode, while the last term is associated to the rotational energy mode. The pure oscillatory phase is characterized by $C_{1}=C_{2}=0$ (on the cylinder), which is a very special class of solution, hardly realized by physically reasonable universes. The escape to inflation and the collapse depend on the relative signs of $C_{1}$ and $C_{2}$, but the smaller are these constants the larger is the time that the orbit spends in the oscillatory phase. Nevertheless it must be stressed that in this oscillating phase the universe has periods of accelerated expansion followed by accelerated contraction, and the escape to inflation actually corresponds to a progressive increase of the inflationary phase.

The next step is to study the behavior of inhomogeneous perturbations of matter $\delta \rho$ and scalar field $\delta \varphi$ in this background. Before presenting the evolution equations for the perturbed quantities, it is important to stress that $\delta \varphi$ will give rise to second order perturbations of the energy of the scalar field, $\delta \rho_{\varphi}$, since $\dot{\varphi}^{(0)} \approx V^{\prime}\left(\varphi^{(0)}\right) \approx 0$, with $\varphi^{(0)} \approx$ constant being the scalar field of the background corresponding to an extremum of the potential. As a consequence, there will be no coupling between $\delta \rho$ and $\delta \rho_{\varphi}$, once the former represents first order deviations.

Introducing the contrast density $\chi=\frac{\delta \rho}{\rho}$, one can show that its evolution equation is given by

$$
\ddot{\chi_{k}}+2 \frac{\dot{l}}{\dot{l}} \dot{\chi_{k}}-\frac{1}{2} \rho \chi_{k}=0 .
$$

Here $\chi_{k}$ is the mode associated to the scalar harmonic basis $Q_{(k)}(\mathbf{x})$ which satisfies $h^{\mu \nu} Q_{(k)}(\mathbf{x})_{, \mu ; \nu}=\frac{k^{2}}{l^{2}} Q_{(k)}(\mathbf{x}), h^{\mu \nu}$ being the projector into the spatial surface, and $k^{2}=n^{2}-1$ with $n \geq 1$ assuming integers values. $\rho=E_{0} / l(t)^{3}$ is the energy density of the background. In the oscillatory phase about $E$ (Eq. (5) with $C_{1}=C_{2}=0$ ), the above equation, after a proper rescaling of the variable $\chi_{k}$, assumes the form

$$
\ddot{\chi_{k}}+(-q+a \cos (2 \omega t)) \chi_{k}=0,
$$

where $q$ is a positive constant, and $|a|<<q$. This is the Mathieu equation inside the parametric region of instability, in which all modes $k$ are equally amplified. If the universe, after this oscillating phase, undergoes to a successful inflation, $l \approx \exp \sqrt{\Lambda / 3} t$, and the equation for all modes $\chi_{k}$ reduces to 


$$
\ddot{\chi_{k}}+\frac{2}{3} \sqrt{\Lambda} \dot{\chi}_{k} \approx 0
$$

As a consequence all modes $\chi_{k}$ will decrease asymptotically, or in other words, all perturbations of matter produced during the oscillatory phase will be washed out in the inflationary regime, and cannot therefore be considered as seeds of structure formation.

Now we consider the evolution of perturbations of the scalar field $\varphi(\mathbf{x}, t)=\varphi^{(0)}(t)+Q_{(k)}(\mathbf{x}) \delta \varphi_{k}(t)$, where the background $\varphi^{(0)}(t) \approx$ constant. The equation of motion for the perturbed modes of the scalar field is

$$
\left(\delta \varphi_{k} \ddot{)}+3 \frac{\dot{l}}{l}\left(\delta \varphi_{k} \dot{)}+\left(V^{\prime \prime}\left(\varphi^{(0)}\right)+\frac{k^{2}}{l^{2}}\right) \delta \varphi_{k}=0 .\right.\right.
$$

Introducing the variable $\delta_{k}=l^{3 / 2} \delta \varphi_{k}$ into the above equation as well as the approximate solution in the oscillatory regime, it follows

$$
\ddot{\delta_{k}}+\left[\left(V^{\prime \prime}\left(\varphi_{0}\right)+\frac{k^{2}}{l_{0}^{2}}\right)-\epsilon\left(\frac{2 k^{2}}{l_{0}^{4}}+\frac{6 \omega^{2}}{l_{0}^{2}}\right) \cos (2 \omega t)\right] \delta_{k}=0,
$$

that is a typical Mathieu equation. As an important property of solutions of this equation is the existence of an instability zone in the parameter space in which the solutions grow exponentially as $\delta_{k} \exp \left(\nu_{k} t\right)$. This instability is known by parametric resonance. For small $\epsilon$, the condition for parametric resonance is given by

$$
\frac{V^{\prime \prime}\left(\varphi^{(0)}\right)}{2 \Lambda}+2 k^{2}=j^{2}+\Delta(\epsilon)
$$

where $j$ is an arbitrary integer and $\Delta(\epsilon)$ is a small adimensional number dependent of $\epsilon$. This means that only the modes with $k$ satisfying the above relation grow exponentially. These modes are determined by the ratio between the mass of the scalar field $V^{\prime \prime}\left(\varphi^{(0)}\right)$, and the vacuum energy of the scalar field. The ratio $\frac{V^{\prime \prime}\left(\varphi^{(0)}\right)}{2 \Lambda}$ is a fundamental parameter in inflationary models; since the resonance mechanism is believed to be general, the order of this parameter could be evaluated through Eq. (11) once the spectrum is experimentally determined. It will be quite ilustrative to choose standard values used for the afore mentioned constants as $V^{\prime \prime}\left(\varphi^{(0)}\right) \approx\left(10^{-12}-10^{-14}\right) m_{p l}^{2}$ and $\Lambda=V\left(\varphi^{(0)}\right) \approx$ $m_{p l}^{2}$, where $m_{p l}$ is the Planck mass. We obtain a very small number of order of $10^{-12}-10^{-14}$ that can be negleted. Thereupon, during the oscillatory phase a selected spectrum of modes, centered about the integers $j^{2}=2 k^{2}$, grow rapidly, whereas the remaining modes are maintained stable. Suppose that, after this regime, the universe escapes to inflation. The equation of motion (9) assumes its usual form as discussed in several works. As we know the perturbed modes that cross the horizon are not affected by microphysical processes, being in this way frozen until they reenter the horizon after inflation. However, due to the mechanism of parametric resonance, the modes which cross the horizon for the first time have not the same amplitude, and are maintained during the kinematical evolution until they reenter the horizon. At the end, the resulting spectrum of adiabatic perturbations is not a flat one, in the sense that there are maxima dictated by Eq. (11). The non-flat spectrum of inhomogeneous fluctuations produced by the above mechanism can explain the hierarchy of scales (galaxies, clusters, etc) as observed in the actual universe.

The authors are grateful to $\mathrm{CNPq}$ and FAPERJ for financial support.

[1] A. Linde, Particle Physics and Inflationary Cosmology, Harwood Academic Publishers, UK (1993).

[2] H. P. de Oliveira, I. Damião. Soares and T. J. Stuchi, Phys. Rev. D 56, 730 (1997). G. A. Monerat, H. P. de Oliveira and I. Damião. Soares, Chaos in Preinflationary Friedamnn-Robertson-Walker Universes, preprint gr-qc/980346 (1998), to appear in Phys. Rev. D.

[3] V. Zanchin, A. Maia Jr., W. Graig and R. Brandenberger, Phys. Rev. D 57, 4651 (1998); Jennie H. Traschen and R. Brandenberger, Phys. Rev. D 42, 2491 (1990); David I. Kaiser, Phys. Rev. D 53, 1776 (1996); L. Kofman, A. Linde and A. A. Starobinsky, Phys. Rev. Lett. 73, 3195 (1994).

[4] M. A. Moser, Commun. Pure Appl. Math., 11, 257 (1958).

[5] A. M. Ozorio de Almeida, N. De Leon, M. A. Metha and C. C. Marston, Physica D 46, 256 (1990); W. M. Vieira and A. M. Ozorio de Almeida, Physica D 90, 9 (1996). 